\title{
Colesteatoma de seno esfenoidal
}

\section{Cholesteatoma Sphenoid Sinus}

\author{
Gabriel Faba $C^{1}$, Ximena Fonseca $A^{1}$, Claudia González $G^{1}$, David Jofré $P^{1}$.
}

\begin{abstract}
RESUMEN
Un colesteatoma de cavidades paranasales es un hallazgo muy raro. Se ha reportado sólo un caso de un colesteatoma primario de seno esfenoidal. Aros casos de colesteatoma que involucren a este seno son debidos a diseminación directa a de un colesteatoma de hueso temporal. Presentamos el caso de un colesteatoma primario de seno esfenoidal, en una mujer de 22 años, que consultó por cefalea periódica. La lesión fue removida a través de un abordaje endoscópico transnasal. 1 diagnóstico fue confirmado por histopatología. No ha presentado recurrencia después de 4 años.
\end{abstract}

Palabras clave: Colesteatoma, seno esfenoidal.

\section{SUMMARY}

A paranasal sinus cholesteatoma is a very rare finding. It has been reported only one case of a primary sphenoidal sinus cholesteatoma; other cases of cholesteatoma involving this sinus are from direct spread of a temporal bone cholesteatoma.

We describe the case of a primary sphenoid sinus cholesteatoma in a 22 year old woman who presented with periodic headache. An endoscopic transnasal sinus approach was used to remove the lesion. The diagnosis was confirmed by histopathology. There has been no recurrence after 4 years of follow up.

Key words: cholesteatoma, sphenoid sinus

\section{INTRODUCCIÓN}

日 origen más común de un velamiento del seno esfenoidal es de origen inflamatorio (sinusitis), aunque el diagnóstico diferencial considera también quistes de retención, mucocele, papiloma, bola fúngica y neoplasias malignas. Sin embargo, un colesteatoma de seno esfenoidal es una entidad extremadamente infrecuente.

Un colesteatoma es una lesión no neoplásica, que produce erosión ósea y puede expandirse hacia áreas vecinas. Histológicamente está compuesto

UDA Otorrinolaringología, Hospital Cínico Pontificia Universidad Católica de Chile. 
por tres diferentes elementos: un centro de queratina, epitelio escamoso y estroma fibroso. Los dos últimos constituyen la matriz del colesteatoma ${ }^{1}$.

Los colesteatomas a menudo se localizan en el hueso. También han sido documentados en riñón, testículo, piel, mama, sistema nervioso central, bóveda craneana, órbita, mandíbula y senos paranasales. En esta última ubicación son muy raros. En 1916 Haeggstrom, fue el primero en reportar un colesteatoma de seno frontal ${ }^{2}$. Desde entonces, otros casos han sido reportados en la literatura. Se localizan más a menudo en el seno frontal y menos frecuentemente en etmoides $y$ senos maxilares ${ }^{3}$.

A continuación presentamos el caso de un colesteatoma de seno esfenoidal.

\section{CASO CLíNICO}

Mujer de 22 años de edad, que consultó por cefalea de un año de evolución. Sin historia de alergias, sinusitis, cirugía nasal ni trauma máxilofacial. En los seis meses previo a la consulta, notó aumento en frecuencia e intensidad de la cefalea $\boxminus$ dolor era bilateral, de intensidad moderada y sensación de presión facial, asociado a rinorrea No presentaba alteraciones neurológicas, descarga posterior ni obstrucción nasal. 日 examen físico fue normal. Se realizó una endoscopía nasal sin hallazgos patológicos.

La Tomografía computada de cavidades paranasales mostró una lesión homogénea dentro del seno esfenoidal, que expandía y desplazaba el tabique del seno esfenoidal, con reacción ósea esclerótica en los bordes y con compromiso del seno etmoidal posterior derecho (Figura 1). Se planteó un mucocele esfenoidal como el diagnóstico más probable.

Se indicó una cirugía endoscópica de seno esfenoidal, el que fue abierto a través de un abordaje transnasal. Dentro del seno se encontraron múltiples láminas de material blanco perlado. 日 aspecto macroscópico era similar a un colesteatoma de oído medio (Figura 2A). En ese momento se pensó que los hallazgos correspondían a una infección por hongos, por lo que se limpió cuidadosamente el seno esfenoidal y se enviaron muestras a biopsia y cultivo. Los últimos fueron todos negativos. $\mathrm{日}$ diagnóstico histopatológico fue colesteatoma de seno esfenoidal (Fgura 2B).

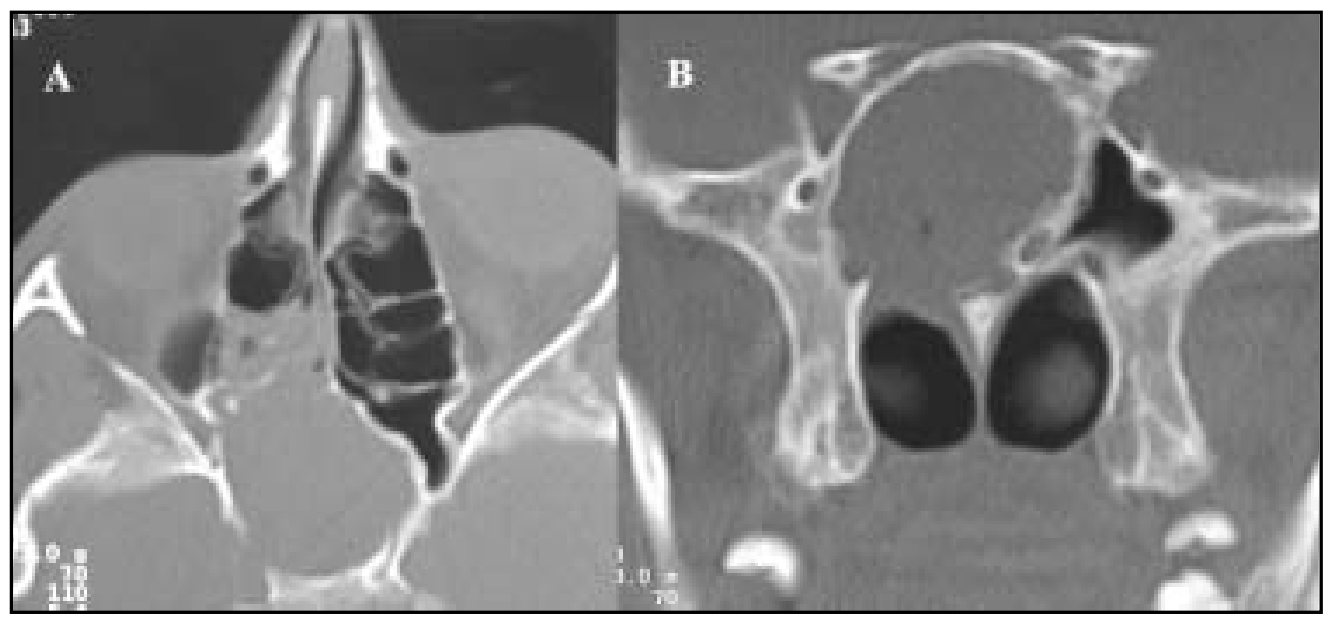

Figura 1. Tomografía computada de cavidades paranasales: lesión homogénea dentro del seno esfenoidal que expande y desplaza el tabique intersinusal, con reacción esclerótica del hueso en los bordes, y compromiso de etmoides posterior derecho. A. Corte axial; B: Corte coronal. 


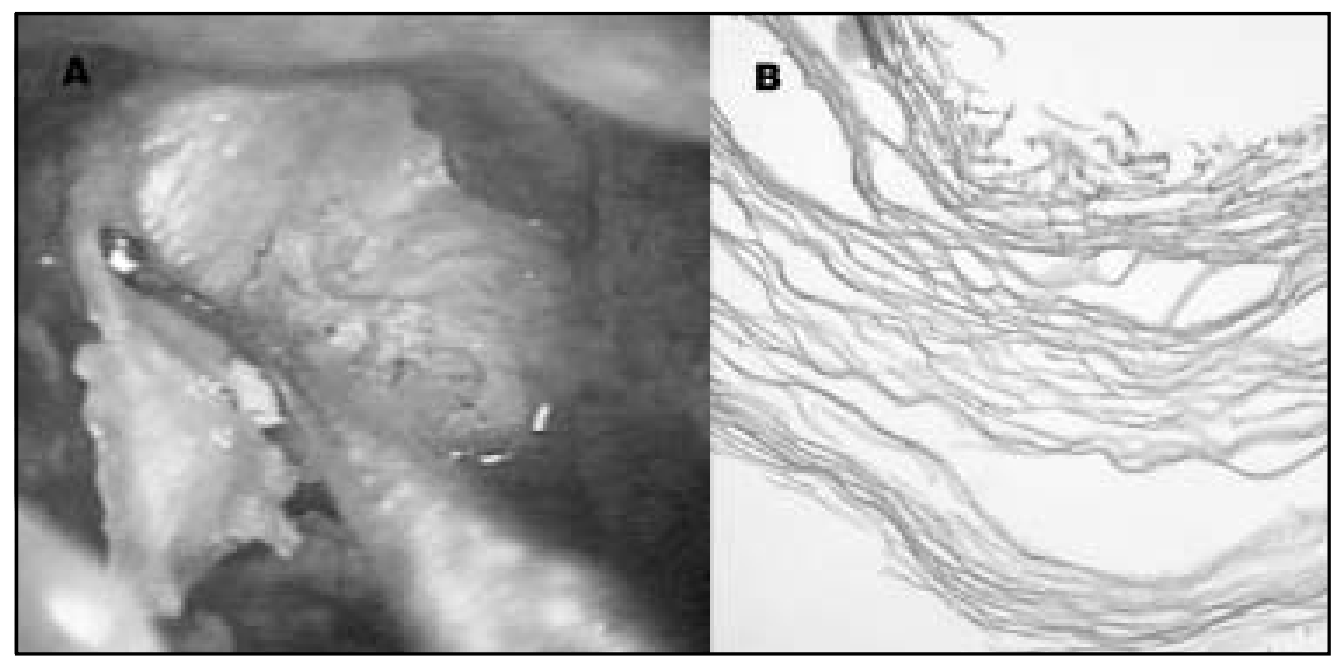

Figure 2. A: Visión endoscópica intraoperatoria del seno esfenoidal. Observe el material laminar blanco perlado dentro del seno. B: Microscopía de luz mostrando láminas de queratina.

Después de la cirugía, la paciente evolucionó favorablemente, sin cefalea ni recurrencias después de 4 años de seguimiento (último control fue en septiembre 2004), pero requiere aseo periódico de la cavidad esfenoidal, debido a la formación de costras.

\section{DISCUSIÓN}

日 hallazgo de un colesteatoma de seno esfenoidal o queratoma es un evento inusual y siempre sorprende al cirujano. Durante los últimos años, se han descrito en la literatura anglosajona, 25 casos de colesteatoma de cavidades paranasales, sólo uno de ellos estaba localizado en forma primaria en el seno esfenoidal. Nosotros presentamos el segundo caso de colesteatoma primario de seno esfenoidal.

日 origen exacto de un colesteatoma de cavidades paranasales es aún incierto. Se han propuesto muchas teorías para esta condición ${ }^{3}$. La explicación más satisfactoria, para nuestro caso, puede ser encontrada en la teoría de $\operatorname{Remar}^{3}$ en que un colesteatoma se desarrolla a partir de explantes de nidos de tejido epidérmico embrionario y como reacción a irritación crónica, como por ejemplo infección, estas células comienzan a crecer. Lateoría de la invasión epitelial, propuesta Habermann, establece que la causa del colesteatoma es la migración de epitelio escamoso queratinizante hacia un área en que usualmente no se encuentra. Procedimientos quirúrgicos o trauma de las cavidades paranasales son fuentes de implantación. Varios casos en la literatura mencionan traumatismo precediendo el hallazgo de un colesteatoma. Esto puede ser válido para un colesteatoma de seno maxilar o frontal que son más susceptibles de trauma facial ${ }^{4}$, pero el seno esfenoidal está bien protegido de este tipo de daño, y en ausencia de cirugía previa en el área (como una hipofisectomía transesfenoidal o cirugía esfenoidal) hace a esta teoría menos probable para explicar el origen de un colesteatoma esfenoidal.

En todos los casos de colesteatoma sinusal, los síntomas fueron causados por la naturaleza expansiva de estas lesiones. $\mathrm{E}$ síntoma más común es dolor que aumenta en intensidad en la medida que la lesión crece. A diferencia de los colesteatomas de seno maxilar o frontal, que también se presentan con deformidad de la frente, exoftalmo, obstrucción nasal o inflamación facial ${ }^{2}$, un colesteatoma esfenoidal puede tener un crecimiento silente, con cefalea como el único síntoma En teoría el efecto de masa podría afectar a cualquie- 
ra de las estructuras relacionadas con el seno esfenoidal, como nervios craneales II, III, IV, V1, V2, dura madre, hipófisis, seno cavernoso y carótida interna Afortunadamente esto no le ocurrió a nuestro paciente, a diferencia del caso reportado por Sani y cols ${ }^{5}$, que se presentó con déficit neurológico. La cefalea es un hallazgo inespecífico, hecho que explica el retardo en el diagnóstico.

日 diagnóstico diferencial de una lesión en el seno esfenoidal incluye patologías no neoplásicas y neoplásicas. En el primer grupo deben considerarse mucocele, bola fúngica, quiste de retención mucosa Las lesiones neoplásicas pueden ser benignas o malignas, como displasia fibrosa, osteoma, papiloma invertido, pólipos, linfoma, adenoma hipofisiario y metástasis. De acuerdo a todos los autores, la tomografía computada de cavidades paranasales es la primera opción de estudio. $日$ hallazgo típico es una lesión expansiva, sin refuerzo, con reacción esclerótica del hueso en los bordes. En nuestro caso los hallazgos tomográficos nos hicieron pensar en un mucocele esfenoidal. La resonancia nuclear magnética es útil en este tipo de lesiones, mostrando una hipointensidad en T1 e hiperintensidad en T24. Aunque ambos exámenes son muy útiles, el diagnóstico de un colesteatoma sinusal rara vez se realiza en el preoperatorio. $\mathrm{日}$ diagnóstico se basa en la histología

日 tratamiento estandar es la cirugía, el objetivo es remover completamente la matriz del colesteatoma, dejando el seno abierto a la cavidad nasal. Una excisión limitada de la matriz estará determinada por el riesgo de lesión de estructuras vecinas, como el nervio óptico o arteria carótida interna $\mathrm{日}$ abordaje quirúrgico depende de la localización del colesteatoma y de la experiencia del cirujano. En nuestro paciente realizamos un abordaje trasnasal del seno esfenoidal con una amplia apertura de la pared anterior del seno esfenoidal, que permitió una limpieza completa de la cavidad durante la cirugía y posteriormente en el seguimiento.

Hay dos casos reportados de colesteatoma no primarios de seno esfenoidal seguidos por un carcinoma ${ }^{6,7}$. Aunque su relación no está bien establecida, creemos que tanto el cirujano, como el paciente deben estar atentos a esta posibilidad y este hecho remarca la importancia del seguimiento.

\section{BIBLIOGRAFÍA}

1. Vaz f, callanan V, leghton S, Risdon Ra. Congenital maxillary sinus cholesteatoma. Int $J$ Pediatr Otorhinolaryngol 2000; 52(3): 283-6.

2. GNGI E GNGI C. Ethmoidal Cholesteatoma. Ann Otol Rhinol Laryngol 1991; 100(5 Pt 1): 424-6.

3. HOPP ML, MoNTGOMERY WW. Primary and se condary keratomas of the frontal sinus. Laryngoscope 1984; 94: 628-32.

4. STORPER IS, NEMMAN AN. Cholesteatoma of the maxillary sinus. Arch Otolaryngol Head Neck Surg 1992; 118(9): 975-7.

5. SANI S, SMITH A, LePPLA DC, ILANGOVAN S, GiICK $R$. Epidermoid cyst of the sphenoid sinus with extension into the sella turcica presenting as pituitary apoplexy: case report. Surg Neurol 2005; 63(4): 394-7.

6. OSBORN DA, WALLACE M. Carcinoma of the frontal sinus associated with epidermoid cholesteatoma. J Laryngol Otol 1967; 18: 102-32.

7. MANIGLIA AJ, VILLA L. Epidermoid carcinoma of the frontal sinus secondary to cholesteatoma. Trans Am Acad Ophtalmol Otholaryngol 1977; 84 (1): 112-5.

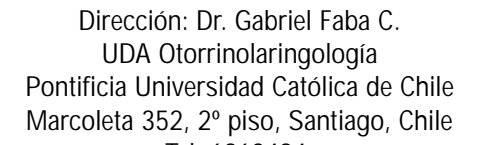

Tel: 6863484. 